\title{
Engineering and design considerations for next-generation snakebite antivenoms
}

Knudsen, Cecilie; Ledsgaard, Line; Dehli, Rasmus Ibsen; Ahmadi, Shirin; Sørensen, Christoffer Vinther; Laustsen, Andreas Hougaard

\section{Published in:}

Toxicon

Link to article, DOI:

10.1016/j.toxicon.2019.06.005

Publication date:

2019

Document Version

Peer reviewed version

Link back to DTU Orbit

Citation (APA):

Knudsen, C., Ledsgaard, L., Dehli, R. I., Ahmadi, S., Sørensen, C. V., \& Laustsen, A. H. (2019). Engineering and design considerations for next-generation snakebite antivenoms. Toxicon, 167, 67-75.

https://doi.org/10.1016/j.toxicon.2019.06.005

\section{General rights}

Copyright and moral rights for the publications made accessible in the public portal are retained by the authors and/or other copyright owners and it is a condition of accessing publications that users recognise and abide by the legal requirements associated with these rights.

- Users may download and print one copy of any publication from the public portal for the purpose of private study or research.

- You may not further distribute the material or use it for any profit-making activity or commercial gain

- You may freely distribute the URL identifying the publication in the public portal 
1 Review

2 Engineering and design considerations for next3 generation snakebite antivenoms

4 Cecilie Knudsen ${ }^{1 *}$, Line Ledsgaard ${ }^{1}$, Rasmus Ibsen Dehli ${ }^{1}$, Shirin Ahmadi ${ }^{1,2}$, Christoffer Vinter

Academic Editor: name

Received: date; Accepted: date; Published: date

\section{Abstract:}

Snakebite envenoming is a devastating Neglected Tropical Disease, the treatment of which has seen relatively little innovation since the invention of antivenom serotherapy in 1894. Current antivenoms have been and continue to be invaluable in saving thousands of lives. However, these medicines are associated with a number of drawbacks pertaining to availability, safety, and efficacy. Fortunately, with the advent of novel methodologies, such as antibody discovery technologies, high-throughput drug discovery approaches, and improved methods for protein engineering, we are starting to see scientific advances in the field. This review presents relevant engineering and design considerations for exploiting these methodologies to develop next-generation antivenoms with improved safety, efficacy, and affordability. The pros and cons of different treatment modalities will be discussed with regards to immunogenicity, the suitability of preclinical efficacy assays, availability of discovery methods, economic viability of production schemes, and possible regulatory approval paths.

Keywords: Next-generation antivenoms; recombinant antivenoms; small molecule toxin inhibitors; toxin-neutralization; monoclonal antibodies; snakebite envenoming; antivenom development; antivenom manufacture.

\section{Introduction}


While the progress of human technology has conquered many diseases, it has left others unaddressed and forgotten. One such neglected disease is snakebite envenoming, which continues to plague vulnerable communities around the world, causing death and disability. Inflicting cytotoxicity, neurotoxicity, hemotoxicity, and other toxicities, snakebite envenoming annually claims somewhere from 81,000 to 138,000 lives, and causes hundreds of thousands of people to live with chronic disablement (Gutiérrez et al., 2017a). Snakebite envenoming disproportionally affects poor, agricultural workers, often the breadwinners of their families, and as such acts to destabilize economically vulnerable population segments (Bawaskar et al., 2017; Chaves et al., 2015; Gutiérrez et al., 2017a; Harrison et al., 2009). Hence, snakebite envenoming is not only a public health issue, but also a socioeconomic challenge in many parts of the world.

Currently, snakebite envenoming is treated with antivenom consisting of polyclonal antibodies or antibody fragments derived from the plasma of hyperimmunized animals (Gutiérrez et al., 2017a). Such antivenoms save countless lives and are therapeutically invaluable. Nonetheless, the administration of heterologous antibodies comes at the inherent risk of immunogenicity, and adverse reactions have been reported both due to this and to inadequate purification procedures for some antivenom products (Burnouf et al., 2004; Chippaux et al., 1998; De Silva et al., 2016; Fry, 2018; León et al., 2013, 2008; LoVecchio et al., 2003; Malasit et al., 1986; Moran et al., 1998; Stone et al., 2013; Sutherland, 1977). Along with the limited availability of antivenoms in certain regions of the world, this has highlighted the need for novel types of antivenom with improved therapeutic properties (Laustsen et al., 2016a). In this review, we seek to discuss engineering and design aspects which should be considered in the pursuit of the next generation of antivenoms. These will be supported by examples of the promising research efforts that have been published in recent years within antibody engineering and drug repurposing.

\section{Efficacy}

One of the most important factors to assess when developing a new antivenom is efficacy. Several assays exist for determining efficacy in the preclinical setting, with the common standard being administering a preincubated mixture of venom and antivenom in an animal model and monitoring survival (see Figure 1A) (Gutiérrez et al., 2017b; World Health Organization, 2018). This assay is suitable for testing antivenoms against lethal, systemic envenomings (e.g. neurotoxicity and venominduced consumption coagulopathy). However, many snake venoms initiate complex pathophysiological effects resulting in various clinical manifestations. Thus, depending on the venom in question, it might be necessary to assess additional parameters, such as nephrotoxicity, cytotoxicity, myotoxicity, and hemotoxicity (Gutiérrez et al., 2017b, 2013). Several tests capable of measuring these and other parameters preclinically have been developed, while tests for parameters such as cardiovascular and renal toxicity are still missing (Gutiérrez et al., 2017b; Williams et al., 2018; World Health Organization, 2010). 
An important issue with the preincubation assay for assessing preclinical efficacy is that it largely negates the influence of pharmacokinetics and toxicokinetics. An (experimental) envenoming therapy could appear to be efficacious when preincubated with venom, but fail to neutralize the venom toxins in a rescue assay, where the antivenom is administered a given period after venom administration and at a different anatomical site (typically intravenously, whereas the venom may be administered subcutaneously, intramuscularly, intraperitoneally, or even intravenously) (see Figure 1B). Lack of efficacy in a rescue assay could thus not only be due to inability of the antivenom antitoxins to strongly bind and neutralize the venom toxins (pharmacodynamics). It could also be due to an inappropriate half-life for the antitoxins, limited volume of distribution of the antitoxins, or because of a rapid onset of irreversible toxic effects caused by venom toxins before the antitoxins are able to intercept and neutralize the venom toxins (pharmacokinetics and toxicokinetics). Additionally, the physiological and immunochemical differences between the model organisms used for the tests and the intended human recipients can render preincubation assays less predictive of clinical efficacy (Rojas et al., 2013; Sanhajariya et al., 2018), although the current assay is accepted as providing good indication for human envenoming. However, the conventional preincubation assay might still be most suitable for molecules with well-characterized pharmacokinetics and for comparing antivenoms based on the same type of scaffold molecules with similar therapeutic properties.

The difference in pharmacokinetics between antibody formats could be hypothesised to make the formats differentially suited for neutralizing different classes of toxins. For instance, smaller antibody formats, which are often assumed to have larger volumes of distribution, might be more suitable for the neutralization of locally acting toxins in distal tissue, such as toxins causing local necrotic effects (Gutiérrez et al., 2003; Seifert and Boyer, 2001). Conversely, larger formats, such as the immunoglobulin $\mathrm{G}$ ( $\mathrm{IgG}$ ) format, might be better suited for neutralizing systemically acting toxins, such as neurotoxins and hemotoxins. The superior half-lives of IgGs might also offer prolonged protection against toxins entering circulation from the bite site at a later time point due to depot effects (Seifert and Boyer, 2001). However, careful consideration should still be given as to which format should be chosen, as studies show that the interplay between pharmacokinetics and pharmacodynamics often makes the situation more complex than what theoretical generalisations might lead one to predict (Gutiérrez et al., 2003). 
A
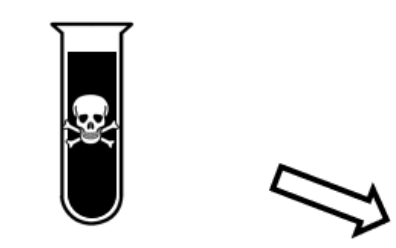

Venom
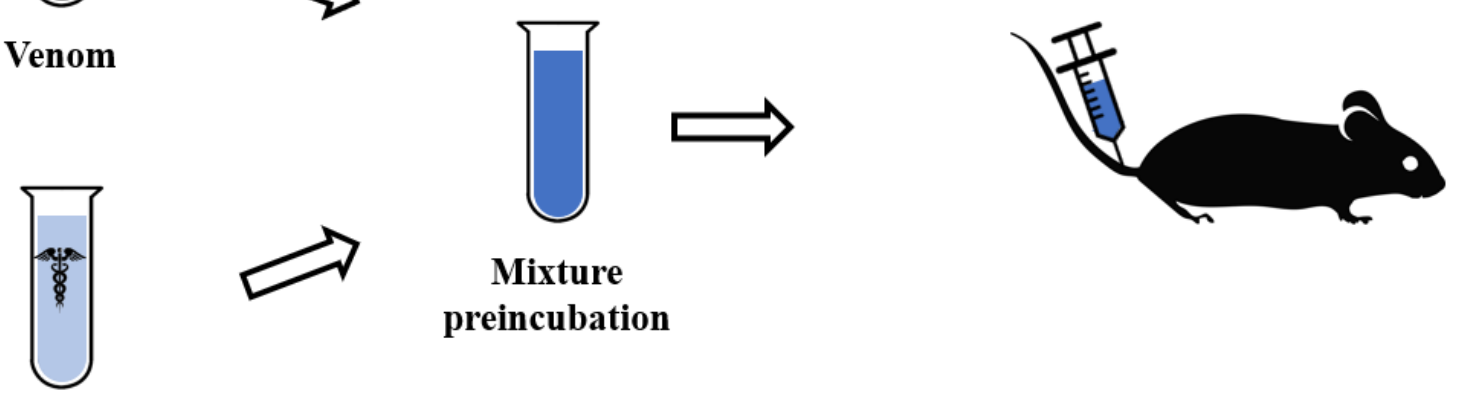

Mixture preincubation

Antivenom

B

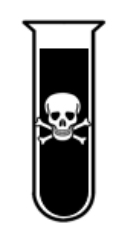

Venom

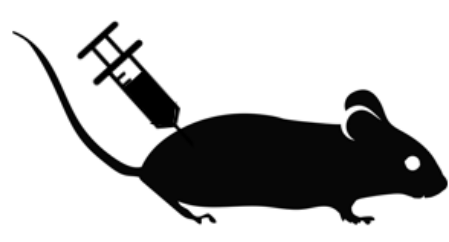

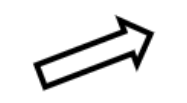

(1)

Figure 1. A) Schematic representation of a preincubation assay, where either whole venom or selected 111 venom components are incubated with the experimental antivenom, prior to administration into the 112 model organism. B) Schematic representation of a rescue assay, in which venom or toxins are injected 113 into the model organism, followed by injection of the experimental antivenom after a delay.

115 Understanding the influence of pharmacodynamics and complementary properties of antibodies in an 116 antivenom is of high importance. However, it is also essential to consider the toxicodynamics and 117 synergistic effects of venom toxins that are to be neutralized with a given novel antivenom. For 118 example, it might be worth considering if it is necessary to neutralize all toxins in a venom to 119 neutralize venom lethality. It has been suggested that by only neutralizing key toxins and/or by 120 disrupting toxin synergism (the phenomenon where a mixture of venom components has a toxicity 121 that is significantly higher than the sum of the toxicities of the components alone), it might be possible 122 to neutralize whole venom lethality (Laustsen, 2016a; Laustsen et al., 2015). This has recently been 123 demonstrated to be the case for Dendroaspis polylepis venom (Laustsen et al., 2018b), as well as it 124 has been demonstrated that antibodies raised against the snake venom metalloproteinase Ecarin from 125 Echis carinatus venom had partial neutralising potential in mice envenomed with E. ocellatus venom 126 (Ainsworth et al., 2018). These findings underline that when employing novel drug development 127 strategies, as well as innovative immunization techniques (Bermúdez-Méndez et al., 2018), it may be 
possible to develop efficacious antivenom products that only target a subset of the venom components.

For several decades, the use of recombinant antibodies has been investigated in the field of antivenom research (Laustsen et al., 2018a, 2016a). Many of these attempts to create novel antivenoms have employed various types of antibody formats. As such, different antibody formats, including nanobodies $\left(\mathrm{V}_{\mathrm{H}} \mathrm{Hs}\right)$, single-chain antibody fragments ( $\mathrm{scFvs}$ ), antigen binding antibody fragments (Fabs and $\mathrm{F}\left(\mathrm{ab}^{\prime}\right)_{2} \mathrm{~s}$ ), and whole monoclonal IgG molecules (see Figure 2A) have demonstrated efficacy in neutralizing snake venoms (Boyer et al., 2013; Brvar et al., 2017; Castro et al., 2014; Julve Parreño et al., 2017; Laustsen et al., 2018b; Richard et al., 2013). In another of these studies, Richard et al. showed that $\mathrm{V}_{\mathrm{H}} \mathrm{Hs}$ isolated from an immune phage display nanobody library, as well as $\mathrm{V}_{\mathrm{H}} \mathrm{H}-$ Fc constructs, effectively neutralized $\alpha$-cobratoxin from Naja kaouthia (Richard et al., 2013). Combined, these studies demonstrate the feasibility of using different types of recombinant antibodies for neutralizing snake venom toxins. This may be unsurprising given that conventional antivenoms based on different antibody formats have been used to successfully treat snakebite victims for more than a century.

A newer approach within development of next-generation envenoming therapeutics focuses on inhibitors of enzymatic toxins, such as phospholipases $A_{2}$ and snake venom proteases. The strength of these small molecule inhibitors lies in their ability to neutralize the activities of multiple different toxins across different species by exploiting the similarities of the catalytic sites of toxin subfamilies. One of the most promising examples of this category of antitoxins is varespladib (LY315920) and its orally available prodrug methyl-varespladib (LY333013) (see Figure 2B). These drugs were originally developed to treat acute coronary syndrome and went through human clinical trials, but were later repurposed for inhibition of phospholipase $\mathrm{A}_{2}\left(\mathrm{PLA}_{2}\right)$ toxins by Lewin et al. (Lewin et al., 2016). In this study, it was demonstrated that mice pretreated or co-injected with varespladib had higher rates of survival when administered with lethal doses of Micrurus fulvius and Vipera berus venom than control mice not treated with varespladib (Lewin et al., 2016). These results were bolstered further, when Bryan-Quirós et al. demonstrated that immediate injection of varespladib at the site of toxin or venom administration could significantly reduce the myonecrotic effects of venom from Pseudechis colletti and Bothrops asper (Bryan-Quirós et al., 2019). In addition to its antimyonecrotic effects, the methylated version of varespladib has shown neutralizing abilities towards lethal doses of neurotoxic venoms from Micrurus fulvius and the Papuan Oxyuranus scutellatus, respectively, in rescue assays in porcine and murine models (Lewin et al., 2018a, 2018b). This could prove especially promising for the treatment of neurotoxic snakebites in Papua New Guinea, where taipans are responsible for a large amount of lethal envenoming cases (Vargas et al., 2011).

Another promising example of the use of small molecule inhibitors for the neutralization of enzymatic toxins is the metalloprotease inhibitor batimastat and its orally available prodrug marimastat (see 
Figure 2B). Like varespladib, batimastat was originally developed for a different indication and later repurposed for snakebite envenoming (Rasmussen and McCann, 1997). Both batimastat and marimastat have been shown to effectively abrogate the hemorrhagic and coagulopathic effects induced by Echis ocellatus venom if administered early and close to the site of venom injection (Arias et al., 2017). Another group of small molecule enzymatic inhibitors is made up by metal chelators, such as EDTA. Several studies show that chelating agents can abrogate toxic effects of snake venom (Ainsworth et al., 2018; Escalante et al., 2017; Howes et al., 2007). However, a common factor for these small molecule inhibitors is that they target only one family of enzymatic toxins. This makes it unlikely that one of them will become a stand-alone therapeutic that can effectively neutralize all toxic effects of a broad range of snake venoms, which typically comprise a multitude of different toxin families. Instead, it seems likely that it may find its use as an adjunctive treatment, as fortification agents for hybrid antivenoms comprising both antibodies and small molecule inhibitors, or as a first aid that can be administered en route to a healthcare facility (Kini et al., 2018). Despite the promise of these and other small molecule inhibitors, to the best of our knowledge, no small molecule inhibitors have ever been marketed for the treatment of snakebite envenoming with the aim of neutralizing the snake toxins.

Other technical avenues for developing improved therapeutics against snakebite envenoming are 185 currently being pursued, including the use of oligonucleotide aptamers and nanoparticles (see Figure 2C and 2D) (Chen et al., 2016; El-Aziz et al., 2017; Karain et al., 2016). More extensive reviews of 187 188 these molecules can be found elsewhere (Knudsen and Laustsen, 2018; Laustsen et al., 2016a, 2016b).
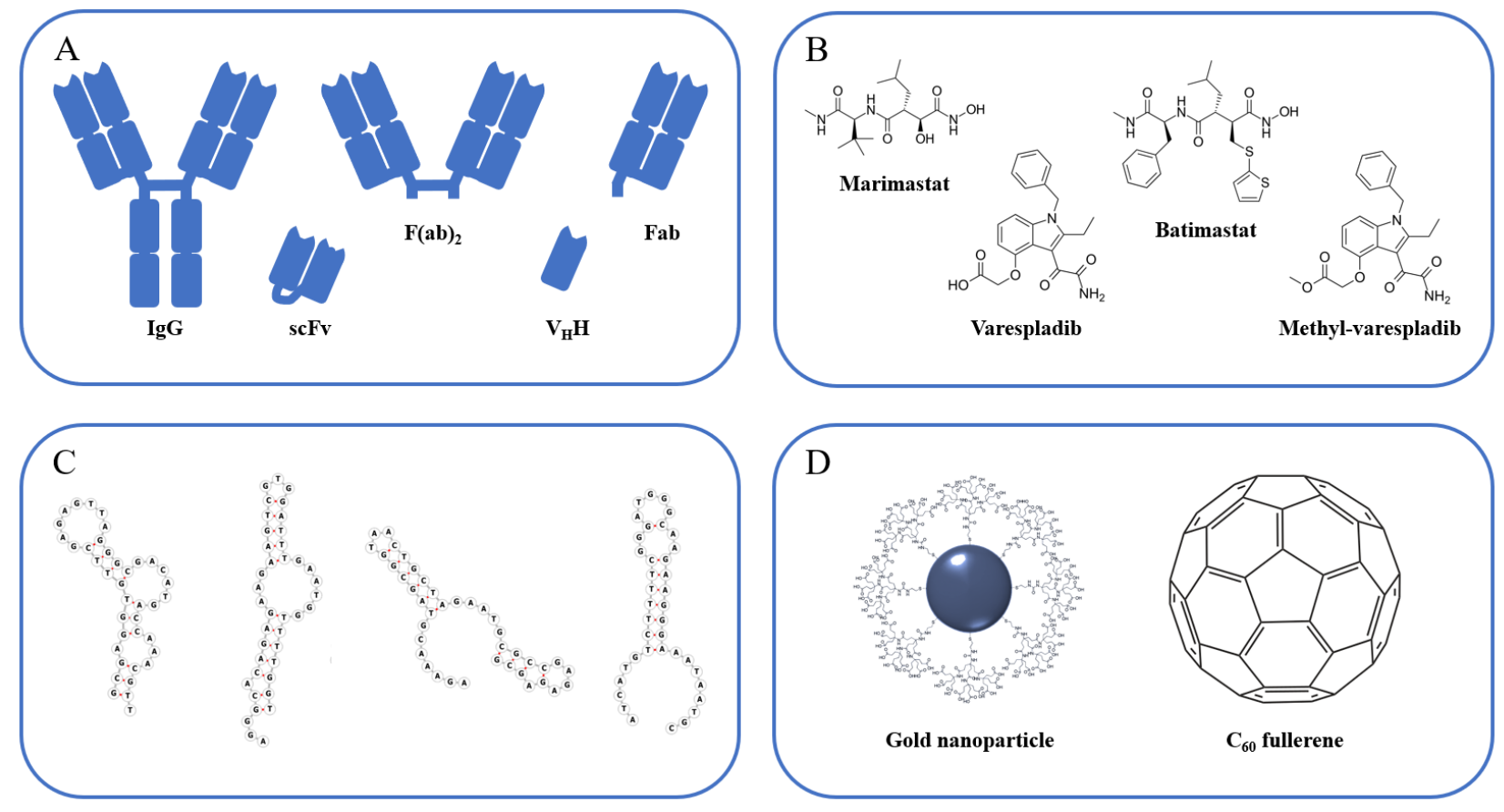

Figure 2. A) Schematic representation of various antibody formats used within antivenom research. 
secondary structures of oligonucleotide aptamers (generated using software described in (Kerpedijev et al., 2015) with sequences from (Chen et al., 2016). D) Schematic representations of two nanoparticles. The representations in this figure are not to scale.

In short, many exciting and promising research results have been reported on the use of various types of molecular compounds for snake toxin neutralization. However, none have progressed to clinical trials. In many cases, it is unknown how the pharmacokinetics and pharmacodynamics of the less commonly studied molecular entities or scaffolds will affect their efficacy in such trials.

\section{Safety}

The safety profiles of current antivenoms often constitute one of their greatest drawbacks, as many antivenoms have been associated with severe adverse reactions (Chippaux et al., 2017). Therefore, the propensity for eliciting adverse effects must be addressed when developing novel antivenom products. For conventional antivenoms, these adverse reactions can be both early-onset, occurring up to 24 hours after administration of antivenom, and late-onset, occurring between 5 and 24 days after antivenom administration (León et al., 2013). Around 20\% of snakebite victims that are administered antivenom experience early-onset adverse reactions (León et al., 2013). However, the incidence rate is related to the antivenom product and can be as high as $88 \%$ and as low as 3\% (León et al., 2013). These reactions can be serious and in rare cases be life-threatening. Early-onset adverse reactions can be divided into two categories; pyrogenic or anaphylactic reactions. The pyrogenic reactions are a direct result of contamination of the antivenom and these reactions can therefore be completely eliminated by implementation of proper product design and good manufacturing practices (GMP) in the production (León et al., 2013). Late-onset serum sickness is a result of an immune response against the heterologous proteins in the antivenom. The actual incidence of these late-onset reactions are not well known, as patients rarely return to the hospitals after treatment (León et al., 2013). In healthcare facilities, which are poorly resourced and staffed by personnel with inappropriate training to tackle such situations, adverse reactions further complicate treatment of snakebite victims (Chippaux et al., 2017). As a result, antivenom is often only administered when clear signs of envenoming manifest themselves (World Health Organization, n.d.), which also has the consequence that antivenom administration may be delayed, allowing the venom toxins to exert their toxic effects for a longer time period. However, when properly manufactured, animal-derived antivenoms having gone through rigorous quality control are associated with few adverse reactions when used to treat animal envenomings in humans (León et al., 2018).

Polyclonal plasma-derived camelid IgGs have been investigated for many years (Harrison et al., 2011), as they have demonstrated a lower propensity towards inducing adverse reactions than polyclonal plasma-derived IgGs of equine and ovine origin (Herrera et al., 2005). It has been suggested that intravenous administration of antivenom based on camelid antibodies might decrease 
the risk of inducing serum sickness and anaphylaxis compared to administration of antivenoms based on equine antibodies (Harrison et al., 2011). However, more recent research pursues recombinant production of camelid single-domain $\mathrm{V}_{\mathrm{H}} \mathrm{H}$ antibody fragments (Prado et al., 2016; Richard et al., 2013). The development of snakebite therapeutics based on murine monoclonal IgG antibodies and antibody fragments has also been attempted (Laustsen et al., 2016a), but is no longer pursued (Laustsen et al., 2018a), as their heterologous nature has proven to cause both early and late adverse reactions, as well as loss of efficacy during repeated use in indications other than snakebite envenoming (Descotes, 2009; Hansel et al., 2010; Laustsen et al., 2018a). To mitigate the risk of immunogenicity, manufacturers of therapeutic antibodies for many other indications increasingly make use of recombinant human antibodies (Grilo and Mantalaris, 2018; Nelson et al., 2010). Human antibodies are compatible with the human immune system, and while they are not entirely devoid of the risk of provoking immunogenicity, they have greatly improved safety profiles compared to humanized, chimeric, and murine antibodies (Hansel et al., 2010; Harding et al., 2010). An additional benefit might be found in their manufacture, as recombinant expression of monoclonal antibodies (mAbs) yields a more defined antibody product (in contrast to isolation of polyclonal antibodies from sera with inherent batch-to-batch variation and undefined composition). This provides the manufacturer with control over which antibodies are produced, unlike when immunization methods are used, and will theoretically enable the antivenom manufacturer to produce antivenoms consisting purely of therapeutically relevant antibodies with proven efficacy. Since some current antivenoms consist of undefined mixtures of antibodies, as few as $5 \%$ of which target snake venom components (Rawat et al., 1994), a shift towards a defined mixture of therapeutically relevant mAbs could potentially lead to development of more efficacious antivenom products. In turn, this may lead to a scenario, where lower doses may be required to treat a given envenoming, thereby possibly lowering both cost of treatment and the risk of adverse effects.

To date, antivenoms comprised of monoclonal IgGs or any other types of recombinant proteins are yet to enter the clinical setting (Laustsen et al., 2018a), but for many other indications there is a rapid increase in market-approved mAbs, especially mAbs of human origin (Grilo and Mantalaris, 2018). This trend may eventually benefit antivenom manufacturers as production of comparatively safe, recombinant antibodies becomes increasingly standardized and economically attractive.

A high number of small non-antibody-based protein scaffolds with different safety profiles are being explored for various indications and may be relevant to investigate in relation to snakebite envenoming (Jenkins et al., 2019; Vazquez-Lombardi et al., 2015). In some cases, it is found that improving the safety profiles of these non-antibody-based protein scaffolds is recommendable. Elimination of $\mathrm{T}$ and/or B cell epitopes is one such strategy employed to decrease immunogenicity and improve safety and efficacy of the protein (King et al., 2014; Mazor et al., 2017; Nagata and Pastan, 2009). Many of non-antibody-based protein scaffolds targeting various indications outside of snakebite have progressed through preclinical trials, and several, like adnectins, affibodies, affilins, 
affitins, anticalins, avimers, DARPins, and Kunitz domains have been tested in Phase I, II, or III clinical trials, respectively (Jenkins et al., 2019; Vazquez-Lombardi et al., 2015). The progression through and completion of clinical trials indicate that some of these scaffolds might be developed and used for therapeutic purposes for a wide range of indications. However, to the extent of our knowledge, the mentioned scaffolds remain clinically untested for the treatment of toxins from snake venoms.

Unlike non-antibody-based protein scaffolds, nanoparticles have been investigated specifically for treating experimental snakebite envenoming. In a recent study, abiotic hydrogel nanoparticles were successfully engineered to randomly associate with and modulate the activity of a diverse array of both $\mathrm{PLA}_{2}$ and three-finger toxin isoforms, inhibiting dermonecrosis caused by the black-necked spitting cobra (Naja nigricollis) (O'Brien et al., 2018). Toxicity studies without venom administration, comparing only the effects of nanoparticle injection to the injection of phosphate buffered saline, provided promising indications of preclinical safety. Mice receiving the nanoparticles intravenously did not exhibit signs of toxicity or behavioral changes. Mice receiving the nanoparticles intramuscularly in the gastrocnemius did not exhibit problems with mobility nor did the mice exhibit a higher plasma creatine kinase activity, which would have indicated myotoxicity. When the nanoparticles were injected intradermally, no macroscopic effects on the skin were observed, and additionally, only normal microscopic appearances were observed in histological assessments subsequent to administration of the nanoparticle via the intravenous, intramuscular, and intradermal routes (O'Brien et al., 2018). In 2016, 51 medicines based on nanoparticles had achieved FDAapproval, and another 77 nanoparticle-based therapeutic candidates were in clinical trials (Bobo et al., 2016). Most of these nanoparticles are of polymeric, liposomal, or nanocrystalline material, but also metallic, micelle-, and protein-based nanomedicines have been approved by regulatory agencies (Bobo et al., 2016). The wide array of nanoparticles which have gained regulatory approval, and the diversity of the indications these nanoparticles target, offer hope that nanoparticles might also find a

Most producers of antibody-based therapeutics outside of the field of snakebite antivenom are moving away from the use of non-human $\operatorname{IgG}$ antibodies. While antivenom researchers are exploring the opportunity to mimic this trend by shifting the focus to nanobodies, human antibodies, and nonantibody-based protein scaffolds, none of this research has yet left the laboratories and entered the clinic. The reports of adverse effects of existing antivenoms highlight one of the greatest drawbacks of the current antivenom generation and thereby one of its greatest and most important opportunities for improvement. Although thorough safety studies of alternative therapeutic modalities for treating envenoming remain scarce, modalities which have proved safe for the treatment of other indications might constitute safer alternatives to the frequently immunogenic antivenoms based on heterologous 


\section{Developability and manufacturing}

311 The process of bringing a pharmaceutical to the market is time-consuming, expensive, and has a success rate of less than $1 \%$ (Kannt and Wieland, 2016). This, in combination with the poor economic incentive to develop new antivenoms, necessitates focusing on tried and tested molecular formats with standardized discovery and production methods. Doing so might minimize the risk of prolonging the process of bringing the drug to the market. Such a prolongation would further increase the costs of the drug development program, forcing the drug developer to charge a high price for the drug to recoup expenditure, and thereby possibly rendering the drug economically unavailable to those who need it (Kini et al., 2018; Laustsen and Dorrestijn, 2018).

Due to their complexity, snake venoms are unlikely to be neutralized by a single molecule, and this makes 'one hit' drugs therapeutically unfeasible. Furthermore, as snake venoms are highly diverse and different across continents, developing a 'universal antivenom' that theoretically would be able to neutralize all key toxins from all snakes across the world seems highly infeasible and irrelevant. However, future antivenoms will most likely be quite complex therapeutic products with mixed compositions of toxin-neutralizing modalities. For the development of an array of molecules that can neutralize different toxins, it could be beneficial to use molecules for which standardized highthroughput discovery protocols exist and where the development and production process can piggyback on experience from other fields. Examples of molecules for which high-throughput discovery protocols have already been developed include aptamers, peptides, and mAbs that can all be selected against toxins in vitro through SELEX (for aptamers) (Tuerk and Gold, 1990) or phage display (for peptides and antibodies) (McCafferty et al., 1990; Smith, 1985). As an example, phage display was employed to select human antibodies against mamba toxins, which were not neutralized by conventional antivenom (Laustsen et al., 2018b). Phage display has additionally been used to discover human scFvs against toxins from Bothrops jararacussu and Crotalus durissus terrificus venom. These scFvs were in vitro reported to inhibit plasma-clotting and prolonged survival of mice injected with lethal doses of the two venoms (Silva et al., 2018).

Repurposing existing drugs might significantly shorten the process of discovery and lead to development of novel adjunctive treatments targeting specific toxin (sub-)families, with one prominent example being the previously mentioned varespladib (Kini et al., 2018; Lewin et al., 2018b, 2016; Wang et al., 2018). Repurposing of such drugs has the potential to lower the cost of treatment as there are much fewer expenses associated with their development.

Another important factor for the cost of a novel antivenom is the cost of its manufacture (Laustsen and Dorrestijn, 2018). For antibody-based antivenoms, recombinant production can be undertaken in of purification, and the scale of the production (Laustsen et al., 2018a). Within large scale 
pharmaceutical antibody production approximately $95 \%$ of therapeutic antibodies are produced in mammalian cell lines such as Chinese Hamster Ovarian (CHO) cells (Jäger et al., 2013; Walsh, 2014). This has prompted antibody researchers to undertake cost-calculations for large scale production of IgG-based antivenoms in mammalian cell lines (see Figure 3C) and lead to the theoretical demonstration that such production methods would likely be cost-competitive with existing manufacturing processes for conventional antivenoms, assuming that oligoclonal expression technologies (such as the Sympress technology) are employed (Laustsen, 2016b; Laustsen et al., 2017; Rasmussen et al., 2012; Wiberg et al., 2006).

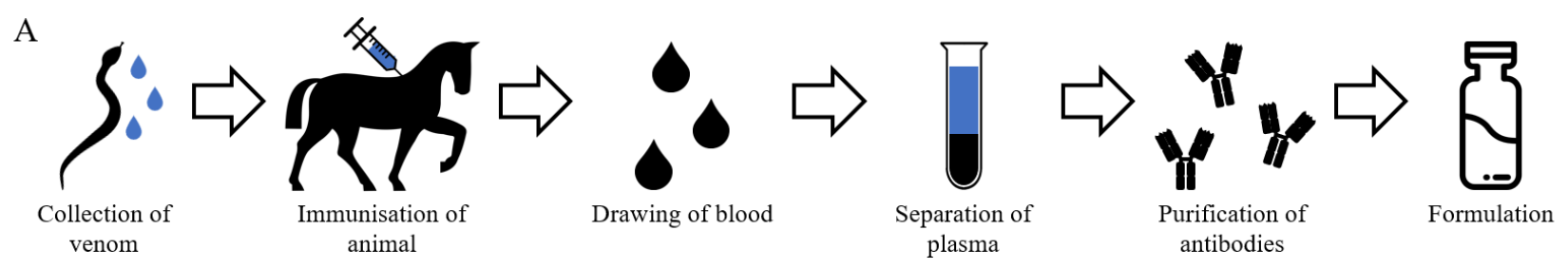

B
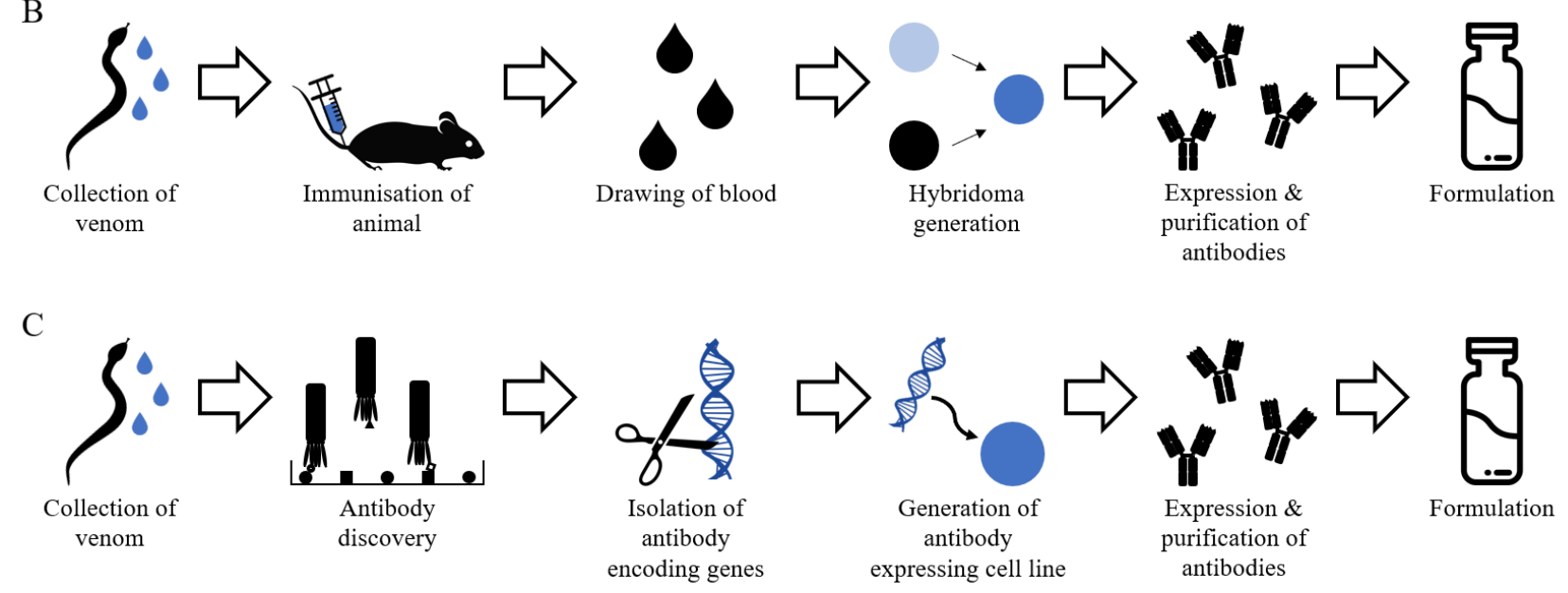

Figure 3. Comparison of antibody generation schemes for antivenoms. A) Antibody generation scheme for many antivenoms in clinical use. B) Generation scheme for antibodies via hybridoma technology. C) Generation scheme for antibodies by phage display technology.

For other types of molecules, such as nucleotide-based aptamers, simple chemical production is often used for small scale synthesis. This allows for cheap production of aptamers required for preclinical testing. However, while some studies present a positive outlook on the large scale cost of manufacture of aptamers (Keefe et al., 2010), studies that thoroughly examine this cost of manufacture are hard to find. It therefore remains unclear what the actual cost of aptamer manufacture is in large scale. It therefore remains to be seen whether this cost is prohibitively high for novel aptamer-based antivenom products that are to reach impoverished victims in the tropics.

Generally, in the pharmaceutical industry, many small molecule therapeutics can be manufactured in large volumes at low cost. However, this does not guarantee that the cost of simultaneous production 
of multiple small molecules for an antivenom will be equally low, as different production facilities operating in parallel will most likely be needed for each of these molecules.

Another important consideration is the downstream processing needed for purification of the active pharmaceutical ingredients after the upstream process. As an example, IgGs and IgG-like proteins can be produced both in mammalian cell cultures and in plants (Julve Parreño et al., 2017; Laustsen et al., 2018b). Even more important than the upstream process is the complex and expensive downstream process, which often accounts for as much as $80 \%$ of the total production cost (Wilken and Nikolov, 2012). Downstream processing of proteins expressed in plants requires harvesting, extraction, clarification of the extract, and purification of the proteins (Lojewska et al., 2016). By comparison, antibodies expressed in cell cultures can be purified using caprylic acid precipitation and/or protein A affinity chromatography (Liu et al., 2010; Morais et al., 2014). This is routinely performed within industrial antibody manufacturing (Hammerschmidt et al., 2014; Liu et al., 2010), with the consequence that these purification methods have been extensively optimized and are highly standardized. Therefore, when choosing a production strategy, it is highly relevant to take the downstream processing, that follows each method of (bio)synthesis, into account.

For a novel antivenom product to be able to replace existing plasma-derived antivenoms, the cost of treatment is of high importance, as these therapies are to be delivered especially to impoverished victims in countries with limited healthcare budgets. To keep costs low, strategies such as choosing a molecule for which standardized discovery and manufacturing protocols exist and considering the cost of both upstream and downstream processing are thus highly relevant. Repurposing of existing drugs could also allow for great reduction of cost of antivenoms' development processes.

\section{Regulatory aspects}

Antivenoms are unusual medicines and have historically not followed the conventional path for regulatory approval. Antivenoms have been used since 1896 in human patients, with limited attention in many clinical trials towards their safety and effectiveness (World Health Organization, 2010). Even to this day, some manufacturers introduce new antivenoms to the market with no conventional clinical evaluation process, and some antivenoms even lack preclinical data to support effectiveness (Williams et al., 2018).

Bringing a drug candidate to the market through the conventional pathway is notoriously expensive and time-consuming (Kannt and Wieland, 2016): The average time from the conclusion of preclinical testing until FDA-approval is granted is 8-9 years, seven of which are spent on clinical trials and 1-2 of which are for FDA review (Reichert, 2010). All in all, the process from drug development to regulatory approval frequently ends up taking over 15 years and costing between USD 3.7 and 11.8 
billion (Kannt and Wieland, 2016); a sum that is prohibitive within antivenom development and marketing (Williams et al., 2018).

Another challenge for current antivenoms is their propensity for inducing adverse effects, which has caused many manufacturers to forfeit phase 1 trials rather than expose healthy individuals to the risk of suffering adverse reactions (Alirol et al., 2017). Fortunately, fundamentally novel types of antivenom (such as recombinant antivenoms based on monoclonal antibodies) are currently being researched and developed (Jenkins et al., 2019; Knudsen and Laustsen, 2018; Laustsen, 2017; Laustsen et al., 2018a, 2016a). These next-generation antivenoms can be designed to have improved safety profiles compared to current antivenoms, as they can be based on less immunogenic modalities. Improved safety could even allow antivenom developers to bring next-generation antivenoms into clinical phase 1 trials. Being able to test novel antivenom products in clinical phase 1 trials has the potential to dramatically improve future envenoming therapies for patients by allowing for early systematic assessment of clinical safety prior to market entry. It can further be speculated, that if the safety of next-generation antivenom products can be demonstrated to be superior compared to existing antivenoms, an opportunity may present itself for earlier antivenom administration, e.g. in an ambulance en route to a treatment facility. Earlier treatment of snakebite envenoming has been correlated with faster recovery and hospital discharge (Anderson et al., 2018; Johnston et al., 2017) and might be especially helpful in the case of rapidly acting toxins, e.g. neurotoxins. Thus, improved safety may very well lead to improved effectiveness for snakebite antivenoms.

Repurposing of approved therapeutics or therapeutic candidates, which have already gone through (some) clinical trials, could be a way to reuse the results from costly clinical safety trials. As an example, varespladib has previously gone through clinical trials, but was arrested in phase 3 due to insufficient efficacy against acute coronary syndrome (Adis, 2011; U.S. National Library of Medicine, n.d.). However, the phase 2 trials of varespladib suggested that six months of daily treatment for acute coronary syndrome with $500 \mathrm{mg}$ active pharmaceutical ingredient generated no adverse effects compared to the placebo treatment. The estimated dose of varespladib required to treat snakebite envenoming in an adult man of $70 \mathrm{~kg}$ has been estimated to be $280 \mathrm{mg}$, which suggests that treatment with varespladib against snakebite would be safe from adverse reactions (Wang et al., 2018). Similarly, the safety of batimastat and marimastat has already been clinically investigated (Parsons et al., 1997; Sparano et al., 2004; Steward, 1999). As such, relying on previous safety

Finally, it is possible that future antivenoms will combine existing molecular formats with new ones. For example, it could be envisioned that traditional antivenoms could be spiked with some of the novel antitoxins discussed here. Small molecule inhibitors with broad neutralizing capacities and assessments of repurposed therapeutics and therapeutic candidates poses another potential path through the regulatory approval system for novel antitoxin molecules.

\footnotetext{
human monoclonal antibodies targeting poorly immunogenic toxins could bolster current antivenoms
} 
(Kini et al., 2018). However, it is difficult to predict how such hybrid products will be viewed by the regulatory authorities, and which regulatory demands hybrid antivenom products may be met with.

In conclusion, next-generation antivenoms might display improved safety and efficacy profiles, which could enable them to go through more conventional clinical trials and regulatory processes. However, funding and practical challenges associated with such trials, as described by Williams et al. (Williams et al., 2018), might still prove problematic or even prohibitive for the completion of clinical trials, and it is difficult to predict how next-generation antivenoms or hybrid antivenoms will be evaluated by the regulatory authorities.

\section{Closing remarks}

An urgent need for improving prevention and treatment of snakebite victims exists. Such an improvement must occur on multiple levels, from the creation of international guidelines and public health policies, over national strategies for snakebite management, to improved therapies that are affordable and available to snakebite victims (Gutiérrez et al., 2014; Harrison and Gutiérrez, 2016). While some countries are reliably served with high-quality antivenom products by several manufacturers, other countries are struck by an antivenom paucity in part due to the poor economic viability of current antivenoms in these markets. This accentuates the need for considering antivenom development and production from an economic perspective. Based on these considerations, it might be relevant to focus on therapeutic modalities which are either inherently broadly specific against certain toxin subfamilies (e.g. some enzymatic inhibitors) or scaffold molecules which can be easily adapted to neutralize multiple targets (e.g. antibodies or similar scaffold proteins). It would further be advantageous to focus on types of molecules, which can be discovered and developed in a parallelized high-throughput fashion and which are manufacturable using standardized large scale, low-cost production processes. Additionally, it might be relevant for the antivenom researcher to focus on modalities with known pharmacokinetics to minimize the risk of failure in preclinical and clinical studies. By allowing these engineering and design considerations to direct the development process for fundamentally novel types of antivenoms, it may one day be possible to bring about safer, more effective, and economically viable antivenom products to snakebite victims in need.

\section{Acknowledgments: This research received no external funding.}

Conflicts of Interest: The authors declare no conflict of interest.

\section{References}

Adis, 2011. Varespladib. Am. J. Cardiovasc. Drugs 11, 137-143. doi:10.2165/11533650-00000000000000

Ainsworth, S., Slagboom, J., Alomran, N., Pla, D., Alhamdi, Y., King, S. i., Bolton, F.M.S., Gutiérrez, J.M., Vonk, F.J., Toh, C.-H., Calvete, J.J., Kool, J., Harrison, R.A., Casewell, N.R., 2018. The 
paraspecific neutralisation of snake venom induced coagulopathy by antivenoms. Commun. Biol. 1. doi:10.1038/s42003-018-0039-1

Alirol, E., Sharma, S.K., Ghimire, A., Poncet, A., Combescure, C., Thapa, C., Paudel, V.P., Adhikary, K., Taylor, W.R., Warrell, D.A., Kuch, U., Chappuis, F., 2017. Dose of antivenom for the treatment of snakebite with neurotoxic envenoming: Evidence from a randomised controlled trial in Nepal. PLoS Negl. Trop. Dis. 11. doi:10.1371/journal.pntd.0005612

Anderson, V.E., Gerardo, C.J., Rapp-Olsson, M., Bush, S.P., Mullins, M.E., Greene, S., Toschlog, E.A., Quackenbush, E., Rose, S.R., Schwartz, R.B., Charlton, N.P., Lewis, B., Kleinschmidt, K.C., Sharma, K., Lavonas, E.J., 2018. Early administration of Fab antivenom resulted in faster limb recovery in copperhead snake envenomation patients. Clin. Toxicol. 0, 1-6. doi:10.1080/15563650.2018.1491982

Arias, A.S., Rucavado, A., Gutiérrez, J.M., 2017. Peptidomimetic hydroxamate metalloproteinase inhibitors abrogate local and systemic toxicity induced by Echis ocellatus (saw-scaled) snake venom. Toxicon 132, 40-49. doi:10.1016/j.toxicon.2017.04.001

Bawaskar, H.S., Bawaskar, P.H., Bawaskar, P.H., 2017. Snake bite in India: a neglected disease of poverty. Lancet 390, 1947-1948. doi:10.1016/S0140-6736(17)32175-X

Bermúdez-Méndez, E., Fuglsang-madsen, A., Føns, S., Lomonte, B., Gutiérrez, J.M., Laustsen, A.H., 2018. Innovative Immunization Strategies for Antivenom Development. Toxins (Basel). 10, 137. doi:10.3390/toxins 10110452

Bobo, D., Robinson, K.J., Islam, J., Thurecht, K.J., Corrie, S.R., 2016. Nanoparticle-Based Medicines: A Review of FDA-approved Materials and Clinical Trials to Date. Pharm. Res. 33, 2373-2387. doi:10.1007/s11095-016-1958-5

Boyer, L. V., Chase, P.B., Degan, J.A., Figge, G., Buelna-Romero, A., Luchetti, C., Alagón, A., 2013. Subacute coagulopathy in a randomized, comparative trial of Fab and F(ab')2 antivenoms. Toxicon 74, 101-108. doi:10.1016/j.toxicon.2013.07.018

Brvar, M., Kurtović, T., Grenc, D., Lang Balija, M., Križaj, I., Halassy, B., 2017. Vipera ammodytes bites treated with antivenom ViperaTAb: a case series with pharmacokinetic evaluation. Clin. Toxicol. 55, 241-248. doi:10.1080/15563650.2016.1277235

Bryan-Quirós, W., Fernández, J., Gutiérrez, J.M., Lewin, M.R., Lomonte, B., 2019. Neutralizing properties of LY315920 toward snake venom group I and II myotoxic phospholipases A2. Toxicon 157, 1-7. doi:10.1016/j.toxicon.2018.11.292

Burnouf, T., Griffiths, E., Padilla, A., Seddik, S., Stephano, M.A., Gutiérrez, J.M., 2004 Assessment of the viral safety of antivenoms fractionated from equine plasma. Biologicals 32, 115-128. doi:10.1016/j.biologicals.2004.07.001

Castro, J.M.A., Oliveira, T.S., Silveira, C.R.F., Caporrino, M.C., Rodriguez, D., Moura-da-Silva, A.M., Ramos, O.H.P., Rucavado, A., Gutiérrez, J.M., Magalhães, G.S., Faquim-Mauro, E.L., Fernandes, I., 2014. A neutralizing recombinant single chain antibody, scFv, against BaP1, A PI hemorrhagic metalloproteinase from Bothrops asper snake venom. Toxicon 87, 81-91. doi:10.1016/j.toxicon.2014.05.017

Chaves, L.F., Chuang, T.-W., Sasa, M., Gutiérrez, J.M., 2015. Snakebites are associated with poverty, weather fluctuations, and El Niño. Sci. Adv. 1, e1500249-e1500249. doi:10.1126/sciadv.1500249

Chen, Y.J., Tsai, C.Y., Hu, W.P., Chang, L. Sen, 2016. DNA aptamers against Taiwan banded krait $\alpha$-bungarotoxin recognize Taiwan cobra cardiotoxins. Toxins (Basel). 8. doi:10.3390/toxins8030066

Chippaux, J.-P., Lang, J., Amadi-Eddines, S., Fagot, I., Rage, V., Le Mener, V., 1998. Clinical safety of a polyvalent $\mathrm{F}(\mathrm{ab}$ ')2 equine antivenom in 223 African snake envenomations: a field trial in Cameroon. Trans. R. Soc. Trop. Med. Hyg. 92, 657-662. 
Chippaux, J.-P., Stock, R.P., Massougbodji, A., 2017. Antivenom Safety and Tolerance for the Strategy of Snake Envenomation Management, in: Snake Venoms. pp. 475-495. doi:0.1007/978-94-007-6410-1_25

De Silva, H.A., Ryan, N.M., De Silva, H.J., 2016. Adverse reactions to snake antivenom, and their prevention and treatment. Br. J. Clin. Pharmacol. 81, 446-452. doi:10.1111/bcp.12739

Descotes, J., 2009. Immunotoxicity of monoclonal antibodies. MAbs 1, 104-111. doi:10.4161/mabs.1.2.7909

El-Aziz, T.M.A., Ravelet, C., Molgo, J., Fiore, E., Pale, S., Amar, M., Al-Khoury, S., Dejeu, J., Fadl, M., Ronjat, M., Taiwe, G.S., Servent, D., Peyrin, E., De Waard, M., 2017. Efficient functional neutralization of lethal peptide toxins in vivo by oligonucleotides. Sci. Rep. 7, 1-9. doi:10.1038/s41598-017-07554-5

Escalante, T., Ovadia, M., Cury, Y., Franceschi, A., León, G., Chaves, F., Gutiérrez, J.M., Rucavado, A., 2017. Inhibition of local hemorrhage and dermonecrosis induced by Bothrops asper snake venom: effectiveness of early in situ administration of the peptidomimetic metalloproteinase inhibitor batimastat and the chelating agent CaNa2EDTA. Am. J. Trop. Med. Hyg. 63, 313-319. doi:10.4269/ajtmh.2000.63.313

Fry, B.G., 2018. Snakebite: When the Human Touch Becomes a Bad Touch. Toxins (Basel). 10, 123. doi: $10.3390 /$ toxins 10040170

Grilo, A.L., Mantalaris, A., 2018. The Increasingly Human and Profitable Monoclonal Antibody Market. Trends Biotechnol. doi:10.1016/j.tibtech.2018.05.014

Gutiérrez, J.M., Burnouf, T., Harrison, R.A., Calvete, J.J., Kuch, U., Warrell, D.A., 2014. A multicomponent strategy to improve the availability of antivenom for treating snakebite envenoming. Bull. World Health Organ. 92, 526-532. doi:10.2471/BLT.13.132431

Gutiérrez, J.M., Calvete, J.J., Habib, A.G., Harrison, R.A., Williams, D.J., Warrell, D.A., 2017a. Snakebite envenoming. Nat. Rev. Dis. Prim. 3, 17063. doi:10.1038/nrdp.2017.63

Gutiérrez, J.M., León, G., Lomonte, B., 2003. Pharmacokinetic-pharmacodynamic Relationships of Immunoglobulin Therapy for Envenomation. Clin. Pharmacokinet. 42, 721-741.

Gutiérrez, J.M., Solano, G., Pla, D., Herrera, M., Segura, Á., Vargas, M., Villalta, M., Sánchez, A., Sanz, L., Lomonte, B., León, G., Calvete, J.J., 2017b. Preclinical evaluation of the efficacy of antivenoms for snakebite envenoming: State-of-the-art and challenges ahead. Toxins (Basel). 9, 1-22. doi:10.3390/toxins9050163

Gutiérrez, J.M., Solano, G., Pla, D., Herrera, M., Segura, Á., Villalta, M., Vargas, M., Sanz, L., Lomonte, B., Calvete, J.J., León, G., 2013. Assessing the preclinical efficacy of antivenoms: From the lethality neutralization assay to antivenomics. Toxicon 69, 168-179. doi:10.1016/j.toxicon.2012.11.016

Hammerschmidt, N., Tscheliessnig, A., Sommer, R., Helk, B., Jungbauer, A., 2014. Economics of recombinant antibody production processes at various scales: Industry-standard compared to continuous precipitation. Biotechnol. J. 9, 766-775. doi:10.1002/biot.201300480

Hansel, T.T., Kropshofer, H., Singer, T., Mitchell, J.A., George, A.J.T., 2010. The safety and side effects of monoclonal antibodies. Nat. Rev. Drug Discov. 9, 325-338. doi:10.1038/nrd3003

Harding, F.A., Stickler, M.M., Razo, J., DuBridge, R.B., 2010. The immunogenicity of humanized and fully human antibodies: Residual immunogenicity resides in the CDR regions. MAbs 2, 256-265. doi:10.4161/mabs.2.3.11641

Harrison, R.A., Cook, D.A., Renjifo, C., Casewell, N.R., Currier, R.B., Wagstaff, S.C., 2011. Research strategies to improve snakebite treatment: Challenges and progress. J. Proteomics 74, 1768-1780. doi:10.1016/j.jprot.2011.06.019

Harrison, R.A., Gutiérrez, J.M., 2016. Priority Actions and Progress to Substantially and Sustainably Reduce the Mortality, Morbidity and Socioeconomic Burden of Tropical Snakebite. Toxins 
Harrison, R.A., Hargreaves, A., Wagstaff, S.C., Faragher, B., Lalloo, D.G., 2009. Snake Envenoming: A disease of poverty. PLoS Negl. Trop. Dis. 3. doi:10.1371/journal.pntd.0000569

Herrera, M., León, G., Segura, Á., Meneses, F., Lomonte, B., Chippaux, J.-P., Gutiérrez, J.M., 2005. Factors associated with adverse reactions induced by caprylic acid-fractionated whole $\mathrm{IgG}$ preparations: Comparison between horse, sheep and camel IgGs. Toxicon 46, 775-781. doi:10.1016/j.toxicon.2005.08.004

Howes, J.M., Theakston, R.D.G., Laing, G.D., 2007. Neutralization of the haemorrhagic activities of viperine snake venoms and venom metalloproteinases using synthetic peptide inhibitors and chelators. Toxicon 49, 734-739. doi:10.1016/j.toxicon.2006.11.020

Jäger, V., Bussow, K., Wagner, A., Weber, S., Hust, M., Frenzel, A., Schirrmann, T., 2013. High level transient production of recombinant antibodies and antibody fusion proteins in HEK293 cells. BMC Biotechnol. 13, 52. doi:10.1186/1472-6750-13-52

Jenkins, T.P., Fryer, T., Ibsen Dehli, R., Jürgensen, J.A., Fuglsang-Madsen, A., Føns, S., Laustsen, A.H., 2019. Toxin neutralization using alternative binding proteins. Toxins (Basel). 11. doi:10.3390/toxins 11010053

Johnston, C.I., Ryan, N.M., O’Leary, M.A., Brown, S.G.A., Isbister, G.K., 2017. Australian taipan (Oxyuranus spp.) envenoming: clinical effects and potential benefits of early antivenom therapy-Australian Snakebite Project (ASP-25). Clin. Toxicol. 55, 115-122. doi:10.1080/15563650.2016.1250903

Julve Parreño, J.M., Huet, E., Fernández-del-Carmen, A., Segura, Á., Venturi, M., Gandía, A., Pan, W. song, Albaladejo, I., Forment, J., Pla, D., Wigdorovitz, A., Calvete, J.J., Gutiérrez, C., Gutiérrez, J.M., Granell, A., Orzáez, D., 2017. A synthetic biology approach for consistent production of plant-made recombinant polyclonal antibodies against snake venom toxins. Plant Biotechnol. J. 1-10. doi:10.1111/pbi.12823

Kannt, A., Wieland, T., 2016. Managing risks in drug discovery: Reproducibility of published findings. Naunyn. Schmiedebergs. Arch. Pharmacol. 389, 353-360. doi:10.1007/s00210-0161216-8

Karain, B.D., Lee, M.K.H., Hayes, W.K., 2016. C60 Fullerenes as a Novel Treatment for Poisoning and Envenomation: A Proof-of-Concept Study for Snakebite. J. Nanosci. Nanotechnol. 16, 7764-7771. doi:10.1166/jnn.2016.12851

Keefe, A.D., Pai, S., Ellington, A., 2010. Aptamers as therapeutics. Nat. Rev. Drug Discov. 9, 537550. doi:10.1038/nrd3141

Kerpedijev, P., Hammer, S., Hofacker, I.L., 2015. Forna (force-directed RNA): Simple and effective online RNA secondary structure diagrams. Bioinformatics 31, 3377-3379.

King, C., Garza, E.N., Mazor, R., Linehan, J.L., Pastan, I., Pepper, M., Baker, D., 2014. Removing T-cell epitopes with computational protein design. Proc. Natl. Acad. Sci. 111, 8577-8582. doi:10.1073/pnas.1321126111

Kini, R.M., Sidhu, S.S., Laustsen, A.H., 2018. Biosynthetic Oligoclonal Antivenom (BOA) for Snakebite and Next-Generation Treatments for Snakebite Victims. Toxins (Basel). 10, 534. doi:10.3390/toxins 10120534

Knudsen, C., Laustsen, A.H., 2018. Recent Advances in Next Generation Snakebite Antivenoms. Trop. Med. Infect. Dis. 3, 42. doi:10.3390/tropicalmed3020042

Laustsen, A.H., 2017. Guiding recombinant antivenom development by omics technologies. N. Biotechnol. doi:10.1016/j.nbt.2017.05.005

Laustsen, A.H., 2016a. Toxin synergism in snake venoms. Toxin Rev. 35, 165-170. doi:10.1080/15569543.2016.1220397

Laustsen, A.H., 2016b. Costing recombinant antivenoms. Nat. Corresp. 538, 41. 
Laustsen, A.H., Dorrestijn, N., 2018. Integrating Engineering, Manufacturing, and Regulatory Considerations in the Development of Novel Antivenoms. Toxins (Basel). 10. doi:10.3390/toxins 10080309

Laustsen, A.H., Engmark, M., Milbo, C., Johannesen, J., Lomonte, B., Gutiérrez, J.M., Lohse, B., 2016a. From Fangs to Pharmacology: The Future of Antivenoms. Curr. Pharm. Des. 9. doi:10.2174/1381612822666160623073438

Laustsen, A.H., Gutiérrez, J.M., Knudsen, C., Johansen, K.H., Méndez, E.B., Cerni, F.A., Jürgensen, J.A., Øhlenschlæger, M., Ledsgaard Jensen, L., Esteban, A.M., Pus, U., Andersen, M.R., Lomonte, B., Engmark, M., Pucca, M.B., 2018a. Pros and cons of different therapeutic antibody formats for recombinant antivenom development. Toxicon. doi:10.1016/j.toxicon.2018.03.004

Laustsen, A.H., Johansen, K.H., Engmark, M., Andersen, M.R., 2017. Recombinant snakebite antivenoms: A cost-competitive solution to a neglected tropical disease? PLoS Negl. Trop. Dis. 11, 1-14. doi:10.1371/journal.pntd.0005361

Laustsen, A.H., Karatt-Vellatt, A., Masters, E.W., Arias, A.S., Pus, U., Knudsen, C., Oscoz, S., Slavny, P., Griffiths, D.T., Luther, A.M., Leah, R.A., Lindholm, M., Lomonte, B., Gutiérrez, J.M., McCafferty, J., 2018b. In vivo neutralization of dendrotoxin-mediated neurotoxicity of black mamba (Dendroaspis polylepis) venom by mixtures of human IgG monoclonal antibodies. Nat. Commun. 9, 3928. doi:10.1038/s41467-018-06086-4

Laustsen, A.H., Lohse, B., Lomonte, B., Engmark, M., Gutiérrez, J.M., 2015. Selecting key toxins for focused development of elapid snake antivenoms and inhibitors guided by a Toxicity Score. Toxicon 104, 43-45. doi:10.1016/j.toxicon.2015.07.334

Laustsen, A.H., Solà, M., Jappe, E.C., Oscoz, S., Lauridsen, L.P., Engmark, M., $2016 b$. Biotechnological trends in spider and scorpion antivenom development. Toxins (Basel). 8. doi: $10.3390 /$ toxins 8080226

León, G., Herrera, M., Segura, Á., Villalta, M., Vargas, M., Gutiérrez, J.M., 2013. Pathogenic mechanisms underlying adverse reactions induced by intravenous administration of snake antivenoms. Toxicon 76, 63-76. doi:10.1016/j.toxicon.2013.09.010

León, G., Segura, Á., Herrera, M., Otero, R., de Siqueira França, F.O., Barbaro, K.C., Cardoso, J.L.C., Wen, F.H., de Medeiros, C.R., Prado, J.C.L., Malaque, C.M.S.A., Lomonte, B., Gutiérrez, J.M., 2008. Human heterophilic antibodies against equine immunoglobulins: assessment of their role in the early adverse reactions to antivenom administration. Trans. R. Soc. Trop. Med. Hyg. 102, 1115-1119. doi:10.1016/j.trstmh.2008.04.038

León, G., Vargas, M., Segura, Á., Herrera, M., Villalta, M., Sánchez, A., Solano, G., Gómez, A., Sánchez, M., Estrada, R., 2018. Current technology for the industrial manufacture of snake antivenoms. Toxicon 151, 63-73. doi:10.1016/j.toxicon.2018.06.084

Lewin, M.R., Gilliam, L., Gilliam, J., Samuel, S., Bulfone, T., Bickler, P., Gutiérrez, J.M., 2018 a. Delayed LY333013 (Oral) and LY315920 (Intravenous) Reverse Severe Neurotoxicity and Rescue Juvenile Pigs from Lethal Doses of Micrurus fulvius (Eastern Coral Snake) Venom. Toxins (Basel). 10, 479. doi:10.3390/toxins10110479

Lewin, M.R., Gutiérrez, J.M., Samuel, S.P., Herrera, M., Bryan-Quirós, W., Lomonte, B., Bickler, P.E., Bulfone, T.C., Williams, D.J., 2018b. Delayed oral LY333013 rescues mice from highly neurotoxic, lethal doses of papuan taipan (Oxyuranus scutellatus) venom. Toxins (Basel). 10, 1-7. doi:10.3390/toxins10100380

Lewin, M.R., Samuel, S., Merkel, J., Bickler, P., 2016. Varespladib (LY315920) appears to be a potent, broad-spectrum, inhibitor of snake venom phospholipase A2 and a possible pre-referral treatment for envenomation. Toxins (Basel). 8. doi:10.3390/toxins8090248

Liu, H.F., Ma, J., Winter, C., Bayer, R., 2010. Recovery and purification process development for monoclonal antibody production. MAbs 2, 480-499. doi:10.4161/mabs.2.5.12645 
Lojewska, E., Kowalczyk, T., Olejniczak, S., Sakowicz, T., 2016. Extraction and purification methods in downstream processing of plant-based recombinant porteins. Protein Expr. Purif. 120, 110-117. doi:10.1016/j.pep.2015.12.018

LoVecchio, F., Klemens, J., Roundy, E.B., Klemens, A., 2003. Serum Sickness Following Administration of Antivenin (Crotalidae) Polyvalent in 181 cases of presumed Rattlesnake Envenomation. Wilderness Environ. Med. 14, 220-221. doi:10.1580/1080-6032(2003)14

Malasit, P., Warrell, D.A., Chanthavanich, P., Viravan, C., Mongkolsapaya, J., Singhthong, B., Supich, C., 1986. Prediction, prevention, and mechanism of early (anaphylactic) antivenom reactions in victims of snake bites. Br. Med. J. (Clin. Res. Ed). 292, 17-20. doi:10.1136/bmj.292.6512.17

Mazor, R., Crown, D., Addissie, S., Jang, Y., Kaplan, G., Pastan, I., 2017. Elimination of murine and human T-cell epitopes in recombinant immunotoxin eliminates neutralizing and anti-drug antibodies in vivo. Cell. Mol. Immunol. 14, 432-442. doi:10.1038/cmi.2015.91

McCafferty, J., Griffiths, A.D., Winter, G., Chiswell, D.J., 1990. Phage antibodies: filamentous phage displaying antibody variable domains. Nature. doi:10.1038/348552a0

Morais, V., Berasain, P., Massaldi, H., 2014. Immunoglobulin Purification by Caprylic Acid. Methods Mol. Biol. 1129, 137-143. doi:10.1007/978-1-62703-977-2

Moran, N.F., Newman, W.J., Theakston, R.D.G., Warrell, D.A., Wilkinson, D., 1998. High incidence of early anaphylactoid reaction to SAIMR polyvalent snake antivenom. Trans. R. Soc. Trop. Med. Hyg. 92, 69-70. doi:10.1016/S0035-9203(98)90959-2

Nagata, S., Pastan, I., 2009. Removal of B cell epitopes as a practical approach for reducing the immunogenicity of foreign protein-based therapeutics. Adv. Drug Deliv. Rev. 61, 977-985. doi:10.1016/j.addr.2009.07.014

Nelson, A.L., Dhimolea, E., Reichert, J.M., 2010. Development trends for human monoclonal antibody therapeutics. Nat. Rev. Drug Discov. 9, 767-774. doi:10.1038/nrd3229

O’Brien, J., Lee, S.-H., Gutiérrez, J.M., Shea, K.J., 2018. Engineered nanoparticles bind elapid snake venom toxins and inhibit venom-induced dermonecrosis. PLoS Negl. Trop. Dis. 12, e0006736. doi:10.1371/journal.pntd.0006736

Parsons, S.L., Watson, S.A., Steele, R.J.C., 1997. Phase I/II trial of batimastat, a matrix metalloproteinase inhibitor, in patients with malignant ascites. Eur. J. Surg. Oncol. 23, 526-531. doi:10.1016/S0748-7983(97)93077-8

Prado, N.D.R., Pereira, S.S., da Silva, M.P., Morais, M.S.S., Kayano, A.M., Moreira-Dill, L.S., Luiz, M.B., Zanchi, F.B., Fuly, A.L., Huacca, M.E.F., Fernandes, C.F., Calderon, L.A., Zuliani, J.P., Pereira da Silva, L.H., Soares, A.M., Stabeli, R.G., Fernandes, C.F.C., 2016. Inhibition of the Myotoxicity Induced by Bothrops jararacussu Venom and Isolated Phospholipases A2 by Specific Camelid Single-Domain Antibody Fragments. PLoS One 11. doi:10.1371/journal.pone.0151363

Rasmussen, H.S., McCann, P.P., 1997. Matrix Metalloproteinase Inhibition as a Novel Anticancer Strategy: A Review with Special Focus on Batimastat and Marimastat. Pharmacol. Ther. 75, 6975. doi:10.1016/S0163-7258(97)00023-5

Rasmussen, S.K., Næsted, H., Müller, C., Tolstrup, A.B., Frandsen, T.P., 2012. Recombinant antibody mixtures: Production strategies and cost considerations. Arch. Biochem. Biophys. 526, 139-145. doi:10.1016/j.abb.2012.07.001

Rawat, S., Laing, G.D., Smith, D.C., Theakston, R.D.G., Landon, J., 1994. A new antivenom to treat eastern coral snake (Micrurus fulvius fulvius) envenoming. Toxicon 32, 185-190. doi:10.1016/0041-0101(94)90107-4

Reichert, J.M., 2010. Metrics for antibody therapeutics development. MAbs 2, 695-700. doi:10.4161/mabs.2.6.13603 
Richard, G., Meyers, A.J., McLean, M.D., Arbabi-Ghahroudi, M., MacKenzie, R., Hall, J.C., 2013. In Vivo Neutralization of alpha-Cobratoxin with High-Affinity Llama Single-Domain Antibodies (VHHs) and a VHH-Fc Antibody. PLoS One 8. doi:10.1371/journal.pone.0069495 Rojas, A., Vargas, M., Ramírez, N., Estrada, R., Segura, Á., Herrera, M., Villalta, M., Gómez, A., Gutiérrez, J.M., León, G., 2013. Role of the animal model on the pharmacokinetics of equinederived antivenoms. Toxicon 70, 9-14. doi:10.1016/j.toxicon.2013.03.013

Sanhajariya, S., Duffull, S.B., Isbister, G.K., 2018. Pharmacokinetics of snake venom. Toxins (Basel). 10. doi:10.3390/toxins10020073

Seifert, S.A., Boyer, L. V., 2001. Recurrence phenomena after immunoglobulin therapy for snake envenomations: Part 1. Pharmacokinetics and pharmacodynamics of immunoglobulin antivenoms and related antibodies. Ann. Emerg. Med. 37, 189-195. doi:10.1067/mem.2001.113135

Silva, L.C., Pucca, M.B., Pessenda, G., Campos, L.B., Martinez, E.Z., Cerni, F.A., Barbosa, J.E., 2018. Discovery of human scFvs that cross-neutralize the toxic effects of $B$. jararacussu and $C$. d. terrificus venoms. Acta Trop. doi:10.1016/j.actatropica.2017.09.001

Smith, G.P., 1985. Filamentous Fusion Phage: Novel Expression Vectors that Display Cloned Antigens on the Virion Surface. Science (80-. ). 228, 1315-1317.

Sparano, J.A., Bernardo, P., Stephenson, P., Gradishar, W.J., Ingle, J.N., Zacker, S., Davidson, N.E., 2004. Randomized phase III trial of marimastat versus placebo in patients with metastatic breast cancer who have responding or stable disease after first-line chemotherapy: Eastern Cooperative Oncology Group Trial E2196. J. Clin. Oncol. 22, 4683-4690. doi:10.1200/JCO.2004.08.054

Steward, W.P., 1999. Marimastat (BB2516): Current status of development. Cancer Chemother. Pharmacol. 43 (Suppl., 56-60. doi:10.1007/s002800051099

Stone, S.F., Isbister, G.K., Shahmy, S., Mohamed, F., Abeysinghe, C., Karunathilake, H., Ariaratnam, C.A., Jacoby-Alner, T.E., Cotterell, C.L., Brown, S.G.A., 2013. Immune Response to Snake Envenoming and Treatment with Antivenom; Complement Activation, Cytokine Production and Mast Cell Degranulation. PLoS Negl. Trop. Dis. 7. doi:10.1371/journal.pntd.0002326

Sutherland, S.K., 1977. Serum Reactions: An Analysis of commercial antivenoms and the possible role of anticomplementary activity in de novo reactions to antivenoms and antitoxins. Med. J. Aust. 2, 841-842.

Tuerk, C., Gold, L., 1990. Systematic Evolution of Ligands by Exponential Enrichment: RNA Ligands to Bacteriophage T4 DNA Polymerase. Science (80-. ). 249, 505-510.

U.S. National Library of Medicine, n.d. Vista-16 trial: Evaluation of Safety and Efficacy of Shortterm A-002 Treatment in Subjects With Acute Coronary Syndrome [WWW Document]. URL https://clinicaltrials.gov/ct2/show/NCT01130246

Vargas, M., Segura, Á., Herrera, M., Villalta, M., Estrada, R., Cerdas, M., Paiva, O.K., Matainaho, T., Jensen, S.D., Winkel, K.D., León, G., Gutiérrez, J.M., Williams, D.J., 2011. Preclinical Evaluation of Caprylic Acid-Fractionated IgG Antivenom for the Treatment of Taipan (Oxyuranus scutellatus) Envenoming in Papua New Guinea. PLoS Negl. Trop. Dis. 5. doi:10.1371/journal.pntd.0001144

Vazquez-Lombardi, R., Phan, T.G., Zimmermann, C., Lowe, D., Jermutus, L., Christ, D., 2015. Challenges and opportunities for non-antibody scaffold drugs. Drug Discov. Today 20, 12711283. doi:10.1016/j.drudis.2015.09.004

Walsh, G., 2014. Biopharmaceutical benchmarks 2014. Nat. Biotechnol. 32. doi:10.1038/nbt.3040

Wang, Y., Zhang, J., Zhang, D., Xiao, H., Xiong, S., Huang, C., 2018. Exploration of the Inhibitory Potential of Varespladib for Snakebite Envenomation. Molecules 23, 391. doi:10.3390/molecules23020391

Wiberg, F.C., Rasmussen, S.K., Frandsen, T.P., Rasmussen, L.K., Tengbjerg, K., Coljee, V.W., 
Sharon, J., Yang, C.-Y., Bregenholt, S., Nielsen, L.S., Haurum, J.S., Tolstrup, A.B., 2006. Production of target-specific recombinant human polyclonal antibodies in mammalian cells. Biotechnol. Bioeng. 94, 396-405. doi:10.1002/bit.20865

Wilken, L.R., Nikolov, Z.L., 2012. Recovery and purification of plant-made recombinant proteins. Biotechnol. Adv. 30, 419-433. doi:10.1016/j.biotechadv.2011.07.020

Williams, D.J., Habib, A.G., Warrell, D.A., 2018. Clinical studies of the effectiveness and safety of antivenoms. Toxicon 150, 1-10. doi:10.1016/j.toxicon.2018.05.001

World Health Organization, 2018. Guidelines for the production, control and regulation of snake antivenom immunoglobulins.

World Health Organization, 2010. WHO guidelines for the production, control and regulation of snake antivenom immunoglobulins. doi:10.1051/jbio/2009043 